\title{
Quantifizierung der Weltumwelt. Zur Geschichte einer Kommunikationsform
}

\author{
Daniel Speich Chassé
}

Eingegangen: 12. Juli 2020 / Angenommen: 1. September 2020 / Online publiziert: 28. April 2021

(C) Der/die Autor(en) 2021

Zusammenfassung Umweltrelevante Problemstellungen sind politisch lange fast ausschließlich in lokalen und nationalen Kontexten bearbeitet worden, obwohl politische Grenzen in naturräumlichen Sachzusammenhängen keine Rolle spielen. Doch heute gehört die globale Umweltproblematik zu den zentralen Herausforderungen der Weltpolitik. Der Beitrag fragt, seit wann und in welcher Form „Umwelt“ als ein alle Menschen betreffendes Problem wahrgenommen wurde, das nach gemeinsamen politischen Lösungen verlangt. Im geschichtlichen Rückblick fällt auf, wie sehr die Umweltpolitik durch numerische Kommunikationen geprägt ist. Ohne Quantifizierung, so die leitende These, hätte es niemals zu dem politisch verhandelbaren Gegenstand „Umwelt“ kommen können, der heute die Schlagzeilen dominiert. Der erste Teil handelt von der Epochenwende um 1970, die für die Konstitution des politischen Problemfelds der Weltumwelt bedeutsam gewesen zu sein scheint. Der zweite Abschnitt greift historisch weiter zurück, um die Genealogie von weltumweltpolitischen Institutionen und Akteuren zu skizzieren. Im letzten Abschnitt wird versuchsweise bestimmt, in welchem Verhältnis die Quantifizierung der Umwelt zu dem Vorgang ihrer Ökonomisierung steht. Dieser Zusammenhang scheint heute so fraglos plausibel, dass beide Prozesse analytisch oft in eins gesetzt werden. Sie haben aber historisch unterschiedliche Taktungen und ihre Verbindung musste aktiv geschaffen werden.

Schlüsselwörter Numerische Kommunikation · Umweltpolitik · Internationale Organisationen · Weltgesellschaft · Geschichte des ökonomischen Denkens

\footnotetext{
D. Speich Chassé $(\square)$

Historisches Seminar, Universität Luzern

Frohburgstr. 3, 6002 Luzern, Schweiz

E-Mail: daniel.speich@unilu.ch
} 


\title{
Quantifying Global Environments. Reflections on the History of a Specific Form of Communication
}

\begin{abstract}
Environmental issues have been viewed almost exclusively from local and national perspectives, despite the fact that political borders are completely irrelevant to those issues. But today, the very fact that the environment is of global concern is ubiquitously accepted. This article focusses on the question of when, and in what form, "the environment" became a global issue that requires common political solutions. The core argument says that without recourse to numbers, the environment could never have risen to its current global political importance. The first part of the article outlines the changes in thinking that occurred around 1970. The second section investigates the genealogy of world societal agents and institutions in the environmental field since the Enlightenment. The final paragraphs address the history of economic thinking. From grasping natural things quantitatively, it seems to be only a small step to turning them into economic assets. But the two historical processes of quantification and "economization" of the environment need to be carefully separated.
\end{abstract}

Keywords Numerical communication · Environmental policy · Iinternational organizations $\cdot$ World society $\cdot$ History of economic thinking

\section{Einleitung}

Die globale Umweltproblematik gehört zu den zentralen Herausforderungen der Gegenwart. Der Beitrag fragt, seit wann und in welcher Form „Umwelt“ als ein alle Menschen betreffendes Problem wahrgenommen und kommuniziert wurde, das nach gemeinsamen politischen Lösungen verlangt. Der Blick auf die internationale Welt politischer Organisationen zeigt, ausgehend von der Umweltkonferenz der UNO von 1972, die Entstehung neuer Akteure und Institutionen, die sich in einer globalen Hinsicht mit Natur befassen. Früher war das nicht so. Wie lässt sich die Entdeckung der Umwelt durch die Weltpolitik historisch verstehen? Was sind wesentliche Bedingungen hierfür gewesen?

Unter der Leitung der norwegischen Politikerin Gro Harlem Brundtland setzte die UNO in den ausgehenden 1980er-Jahren eine hochkarätige Kommission zum weltweiten Zusammenhang von Entwicklung und Umwelt ein. Ihr Bericht machte den alten Begriff der „Nachhaltigkeit“ für die weltumweltpolitische Kommunikation zentral (Borowy 2014). Als bedeutsam erwies sich dann der sogenannte „Erdgipfel“, der 1992 in Rio de Janeiro stattfand. Delegationen von fast allen UNO-Mitgliedsländern und einer fast unüberschaubaren Anzahl von Nichtregierungsorganisationen, die sich teils sehr lokal, teils weltweit für den Schutz der Umwelt einsetzen, fanden sich in der brasilianischen Stadt zu der größten bis dahin je ausgerichteten Weltkonferenz zusammen. In Rio kam es 1992 zu einigen Organisationsinnovationen, die an bestehende Körperschaften wie der International Union for the Conservation of Nature (IUCN, gegründet 1948) oder dem United Nations Environmental Program (UNEP, gegründet 1972) weitgehend vorbeigingen. Mit Rückgriff auf das bereits 1988 ent- 
standene Intergovernmental Panel on Climate Change (IPCC), einer zunächst fast rein wissenschaftsinternen Kommunikationsplattform, entstand im Zusammenhang mit der Konferenz von Rio 1992 das Rahmenübereinkommen der Vereinten Nationen über Klimaänderungen (United Nations Framework Convention on Climate Change, UNFCCC) mit Sitz in Bonn. Die wichtigsten Ergebnisse der Umweltkonferenz von Rio waren die Schärfung des Begriffs „Biodiversität“ (Boenigk und Wodniok 2014, S. 4) und vor allem die Neufokussierung der weltgesellschaftlichen Kommunikation auf die Klimafrage, die dann in einer Folgekonferenz 1997 in Kyoto zu einem diplomatisch verbindlichen Protokoll führte (Meckling 2011, S. 75 ff.). Seither ist Weltumweltpolitik praktisch gleichbedeutend mit Klimaschutz, mit Biodiversität am Rande.

Die folgenden Ausführungen drehen sich um drei Beobachtungen. Erstens geht es um die Tatsache, dass das globale Phänomen ,Umwelt“ erst in den 1970er-Jahren als ein alle Menschen betreffendes Problem erkannt wurde, das nach gemeinsamen politischen Lösungen verlangt. Zweitens scheint in diesem Verständigungsprozess die Kommunikation von Zahlen wichtig gewesen zu sein. Im geschichtlichen Rückblick fällt auf, wie sehr die Umweltpolitik durch numerische Kommunikationen geprägt ist. Ohne Zahlen, so die leitende These, hätte es niemals zu dem politisch verhandelbaren Gegenstand „Umwelt“ kommen können, der heute die Schlagzeilen dominiert. Es gibt eine lange Geschichte der Quantifizierung von Naturphänomenen, die bis ins Zeitalter der Aufklärung zurückreicht. Wenn in den vergangenen Jahrhunderten Umweltverhältnisse zu politischen Themen wurden, spielten Praktiken des Messens, des Zählens und des numerischen Vergleichens kommunikativ stets eine wichtige Rolle, gerade wenn dabei die Verhältnisse des ganzen Planeten thematisiert wurden. Und drittens ist nach dem Zusammenhang zu fragen, der zwischen der Quantifizierung und der Ökonomisierung von Umweltverhältnissen in der Weltgeschichte besteht.

Der erste Teil handelt von der Epochenwende um 1970, die für die Konstitution des politischen Problemfelds der Weltumwelt bedeutsam gewesen zu sein scheint. Seither ist Umwelt zu einem globalen Thema geworden, wobei die Reduktion der Umweltfrage auf das Klimaproblem hervorsticht. Der zweite Abschnitt greift historisch weiter zurück, um die Genealogie von weltumweltpolitischen Institutionen und Akteuren zu skizzieren. Es geht um die spezifische Bedeutung von Zahlen im weltpolitischen Kommunikationsprozess, an dem lokal und international im Verlauf des 20. Jahrhunderts immer mehr Akteure teilgenommen haben. Im letzten Abschnitt wird versuchsweise bestimmt, in welchem Verhältnis die Quantifizierung zu dem Vorgang der Ökonomisierung steht. Dieser Zusammenhang scheint heute so fraglos plausibel, dass beide Prozesse analytisch oft in eins gesetzt werden. Sie haben aber historisch unterschiedliche Taktungen und ihre Verbindung musste aktiv geschaffen werden.

Umweltrelevante Problemstellungen sind politisch lange fast ausschließlich in lokalen und nationalen Kontexten bearbeitet worden, obwohl politische Grenzen in naturräumlichen Sachzusammenhängen keine Rolle spielen. Nichtmenschliche Akteure halten sich nicht an Staatsgrenzen und haben sicher auch keinen Begriff von Nation (allenfalls von Heimat im Sinne eines Brutplatzes oder einer ökologischen Nische). Anders sieht es bei menschlichen Akteuren aus, die in Hinsicht auf 
natürliche Zusammenhänge erst gegen Ende des 20. Jahrhunderts zu einer global organisierten Handlungsweise fanden. Obschon gerade Umweltverhältnisse inhärent global sind, dauerte es lange, bis sie zu einem weltweit relevanten Thema gemacht wurden, das eine internationale Lösung erfordert.

Bis etwa 1970 gab es zwar wissenschaftliche Institutionen, die die Weltumwelt zu erfassen versuchten, aber kaum wirkmächtige politische Akteure, die sich in einer globalen Perspektive um Umweltprobleme kümmerten. Das hat mit dem Umstand zu tun, dass bis in die 1970er-Jahre hinein auch der Gegenstand selbst, die Umwelt, begrifflich nur schwach konturiert blieb. Seither entwickelte sich der Umweltschutz zu einer Kernproblematik in der Weltverständigung - ungefähr analog zum kommunikativen Bedeutungszuwachs der Menschenrechte. Dabei spielten Zahlen eine zentrale Rolle. Die Quantifizierung von Natur ist der Kern der Entstehung der modernen Naturwissenschaften, die eine wichtige Rolle als Informationsagentur für politische Entscheide erlangten. Neben anderen numerischen Indikatoren wurde in den letzten Jahren der Vergleich von $\mathrm{CO}_{2}$-Ausstößen zur wichtigsten Kommunikationsform über Nationsgrenzen und andere global wirksame Trennlinien hinweg, wie Rasse oder Geschlecht. Mit Blick auf die Erhaltung einer möglichst großen Vielfalt von Arten hat sich überdies unter dem Stichwort der Biodiversität eine weitere quantifizierende, numerische Kommunikation etabliert. Wegen ihrer großen Bedeutung dienen diese beiden Felder, die Klimapolitik und die Biodiversitätspolitik, im Folgenden als Beispiele dafür, über das Phänomen der Quantifizierung der Umwelt vor dem Hintergrund einer „Weltvergesellschaftung“ nachzudenken.

Seit den 1960er-Jahren stieg die Ökonomie als Sozialwissenschaft im Anschluss an den Prozess der Quantifizierung von Umweltverhältnissen zu einer Leitwissenschaft auf. Ihr Vorbild waren die Naturwissenschaften, die zur gleichen Zeit vermehrt auch nach den durch Menschen verursachten Fehlgängen fragten, um den politischen Akteuren Handlungsanweisungen zur Behebung von Umweltschäden anzubieten. Ungefähr ebenso lang wird in den Wirtschaftswissenschaften über die günstigsten umweltpolitischen Interventionsmodelle debattiert. Die Umweltökonomie ist in der globalen Verständigung seither ebenso wichtig geworden wie die Umweltnaturwissenschaften, obwohl sie im Vergleich zu den Naturwissenschaften für eine solche Zuständigkeit nicht unbedingt prädestiniert ist.

So hat sich z. B. der Politikbereich der Biodiversität ganz wesentlich als eine Buchhaltungspraxis etabliert. In einer weltweiten Bilanz konsolidiert z. B. die International Union for the Conservation of Nature (IUCN) lokale Zählergebnisse, aus denen sich eine rote Liste der bedrohten Arten ablesen lässt (Schleper 2019). Die Möglichkeitsbedingung für eine solche Operation ist eine stabile Kategorisierung des Lebens nach Spezies, die klar voneinander abgrenzbar sind. Exemplarisch lässt sich dann für begrenzte Territorien (z. B. $1 \mathrm{~m}^{2}$ ) die Anzahl verschiedener Arten sowie die Anzahl von Exemplaren einer einzelnen Art auszählen und auf Nationalstaaten, geophysikalisch abgrenzbar scheinende Landschaften, Kontinente oder sogar die ganze Welt hochrechnen. Der Vergleich solcher Listen auf einem Zeitstrahl erlaubt in einem weiteren Abstraktionsschritt Aussagen über die quantitative Zunahme oder Abnahme von Diversität. Im Rahmen der UNESCO entstand Ende der 1960er-Jahre und motiviert durch diese Informationen ein umfassendes Programm mit dem Namen „Man and Biosphere“. Es führte zu weltweiten Indikatoren der Biodiver- 
sität und zur Bestimmung von besonders biodiversen Biotopen mit entsprechend hohem Schutzwert, die wiederum, auf sehr hohem Abstraktionsgrad, miteinander vergleichbar erschienen. Heute gilt ein hohes Maß an Biodiversität als Indikator für resiliente Naturverhältnisse. Die diesbezügliche Buchhaltungspraxis ist so weit ausdifferenziert, dass analog zur volkswirtschaftlichen Gesamtrechnung von einer in Geldwerten quantifizierbaren „Dienstleistung“ von Ökosystemen an die Volkswirtschaften gesprochen werden kann (Fatheuer et al. 2015; Schmelzer 2016).

$\mathrm{Zu}$ fast noch größerer Perfektion ist die Buchhaltungspraxis in der Klimapolitik gelangt, wobei man es hier allerdings mit einem ganzen Arsenal von numerischen Kommunikationspraktiken $\mathrm{zu}$ tun hat, die teilweise unterschiedlich funktionieren und nicht ohne Weiteres kompatibel sind. Die Tatsache eines systemisch interagierenden Weltklimas wird seit dem 19. Jahrhundert beobachtet. Diese Beobachtung konnte konkret nur durch die Verwandlung lokaler Phänomene (Wind, Niederschlag, Temperatur) in miteinander kompatible Zahlenreihen erfolgen. Hinzu trat spät im 20. Jahrhundert die Analyse der molekularen Zusammensetzung von Lufteinheiten und deren Vergleich im Raum und in der Zeit. Über eine Vielzahl von Übersetzungsschritten, die im Detail hier nicht nachgezeichnet werden können, resultierte aus dieser Zählarchitektur die kognitive Möglichkeit einer Luftqualitätsbuchhaltung. Ausgehend von der Tatsache, dass der Anteil von $\mathrm{CO}_{2}$-Partikeln pro Volumeneinheit der Luft entscheidend ist für die Speicherung von Sonnenenergie auf der Erde und daher (zusammen mit anderen treibhauswirksamen Molekülen) zur Erderwärmung beiträgt, hat sich die weltumweltpolitische Kommunikation auf $\mathrm{CO}_{2}$-Emissionshandelssysteme geeinigt. Hier ermöglicht die Grundoperation der buchhalterischen Quantifizierung abstrakt die Allokation von Kosten und Nutzen im globalen Raum der Weltgesellschaft.

Die Wissenschaftsgeschichte argumentiert mit guten Gründen, dass die kontrollierten Quantifizierungspraktiken den Gegenstand der Biodiversität erst hervorgebracht hätten, und ihn keineswegs einfach abbilden, obwohl letzteres das Selbstverständnis der beteiligten Akteure sei (Devictor und Bensaude-Vincent 2016). Und auch in der Klimapolitik scheinen Praktiken des Zählens den so beobachteten Gegenstand erst erzeugt zu haben (Heymann 2019). Schon lange bevor Umwelt als ein weltweit relevantes Thema entdeckt wurde, machten Akteure, wie z. B. Alexander von Humboldt, die Natur des Planeten zu einem Gegenstand von weltumspannenden numerischen Kommunikationen. So plausibel heute der Weltbezug der Umweltfragen ist, so fraglich bleibt seine Historie.

Wenn man Umwelt global versteht, muss sie eine weltgeschichtliche Vergangenheit haben. Möglichst lange Zeitreihen (z.B. aus arktischen Eisbohrkernen) sollen den Einfluss der Menschheit auf die geophysikalischen Interaktionen belegen. Die heute gängige Annahme ist, es habe immer schon eine Umwelt gegeben. Das zeigt die reiche geistes- und sozialwissenschaftliche Diskussion rund um das Stichwort „Anthropozän“, auf die hier nicht eingegangen werden kann. Vielmehr gilt die Aufmerksamkeit dem geschichtlichen Voraussetzungsreichtum dieser Annahme. Faktisch fehlen bis ins späte 20. Jahrhundert schriftliche Überlieferungen darüber, dass sich Menschen schon früher politisch mit globalen Naturbezügen auseinandergesetzt haben. Um diese Schwierigkeit erfassen zu können, wird hier der ahistorische Begriff einer „Weltumwelt“ gesetzt, der in keinen geschichtlichen Quellen zu fin- 
den ist. In der heutigen Bedeutung einer Weltumwelt hat das Wort „Umwelt“ in der politischen Semantik vor etwa 1970 nicht existiert (Warde et al. 2019). Vielmehr sprach man vom „Kosmos“ oder fand andere Konzepte und meinte einen individuell gefühlten Gesamtzusammenhang allen Lebens. Von der Existenz einer vorsozialen „Weltumwelt“" auszugehen, ist ein methodischer Kniff, um den Sachverhalt künstlich in die Vergangenheit zu verlängern. Um die Genealogie der umweltbezogenen globalen Vergesellschaftung sichtbar zu machen, wie es hier versucht wird, ist der konkrete Inhalt von Weltumwelt stets in der Schwebe zu halten. Man muss ihn als das Ergebnis früherer sozialer Interaktionen ausweisen, anstatt ihn zu verdinglichen.

\section{Konturen der Weltumweltpolitik im Epochenbruch der 1970er-Jahre}

Umweltverhältnisse sind inhärent global, wenn man an die systemischen Zusammenhänge des Wetters, der Luft- und Wasserkreisläufe oder auch an die geophysikalische Tektonik denkt. Ihre Globalität ist durch menschliche Wirkungen noch verstärkt worden. Unter dem Stichwort eines „ökologischen Imperialismus“ wurde argumentiert, von Europa aus hätten sich seit dem 16. Jahrhundert eine Reihe von krankheitserregenden Mikroben verbreitet, an denen viele Menschen starben, wodurch die Möglichkeit des kriegerischen Widerstands gegen die europäische Expansion schwand. Die Portugiesen, Spanier, Niederländer und Briten hätten überdies Nutztiere und Pflanzen auf ihren Reisen mitgenommen und so unwissentlich ganze Landstriche im Norden und Süden des amerikanischen Doppelkontinents, im südlichen Afrika und zu einem gewissen Grad auch in Australien und Neuseeland zu neuen Ökosystemen umgeschaffen, die denen Europas immer ähnlicher wurden (Crosby 1991). Zugleich brachte die Expansionswelle aus Europa eine neue Form der Naturausbeutung in viele Teile der Welt (und befeuerte sie zugleich), die stark auf die Gewinnung von Kapital und dessen Akkumulation ausgerichtet war (Beckert 2014). Zur Sicherung dieser Machtverhältnisse entstanden nach Zentren (Europa) und Peripherien (Rest der Welt) organisierte politische Strukturen mit großer geografischer Reichweite (Wallerstein 1986).

Aber erst ganz am Ende dieses langen Prozesses der politischen Weltveränderung und der ökologischen Weltangleichung, d.h. im letzten Drittel des 20. Jahrhunderts, entstanden auch ein globales Politikverständnis und entsprechende Institutionen, um die Weltumwelt zu regieren (Selcer 2018). Dabei gewann ein Ergebnis der europäischen Kolonialexpansion, nämlich die weltwirtschaftliche Ungleichheit zwischen den reichen Industrieländern und den armen Staaten des ,globalen Südens“, eine große Bedeutung und hat diese bis heute behalten (Macekura 2015). Wie lässt es sich erklären, dass es so lange gedauert hat, bis weltgesellschaftliche Institutionen entstanden, die potenziell in der Lage sind, allen lokalen und nationalen Akteuren klare Handlungsvorgaben im Feld der Umweltpolitik zu machen? Welche Rolle spielte dabei die Quantifizierung?

Konkret war wohl der Umstand wichtig, dass es lange keinen generalisierten Begriff für die Naturverhältnisse von menschlichen Gesellschaften gegeben hat (Warde et al. 2019). Begriffsgeschichtlich zeigt sich eine enorme Verschwommenheit der Konzepte. Natur ist als Rahmen der Vergesellschaftung - so wie die Vergesellschaf- 
tung selbst - lange ein lokales Phänomen gewesen. Das Wetter, das Wachstum der Pflanzen und die Interaktion der Tiere äußern sich in der Alltagsbeobachtung in erster Linie vor Ort. Die vielen Phänomene, die heute als Weltumwelt zusammengefasst sind, zeigten ihren systemischen Zusammenhang lange nicht offen. Die Konzipierungsweisen des Umweltverhältnisses divergierten entsprechend dieser ganz heterogenen Kontingenzerfahrungen stark. Darüber hinaus war es für viele nichteuropäische Gesellschaften nie notwendig, überhaupt einen Begriff für Natur oder die natürliche Umwelt zu entwickeln. So schreibt ein Autor aus Gabun in einer Anthologie, die die Begrifflichkeit der Moderne weltweit vergleicht (Emane 2013, S. 193):

Dans les différents langues du Gabon, il n'existe pas de mot correspondant à la nature. Il apparaît même tentant de reprendre l'idée d'Emile Durkheim selon laquelle la nature ne peut pas exister dans une société qui ne connaît pas la science, ou qui n'appréhende pas l'opposition entre la culture et la nature. En fang, enyeng est le terme qui se rapproche le plus de l'idée que l'on se fait de la nature dans les sociétés modernes. Sa traduction la plus appropriée est la ,vie‘, mais enyeng évoque aussi l'existence, voire le monde. ${ }^{1}$

In Anbetracht dieser semantischen und inhaltlichen Offenheit erstaunt es nicht, dass die Vielfalt der natürlichen Phänomene und die Diversität des soziokulturellen Umgangs mit ihnen die Entstehung einer Weltumweltpolitik historisch erschwert haben.

Die Vielfalt oder Unbestimmtheit des Sachzusammenhangs Weltumwelt war auch noch das Hauptproblem an der ersten Umweltkonferenz der UNO, die 1972 in Stockholm stattfand. Erstmals fanden unter dem Dach der Vereinten Nationen Menschen aus vielen Ländern in der schwedischen Hauptstadt zusammen, um über die Bedrohung der natürlichen Lebensbedingungen zu debattieren, nachdem seit der letzten Dekolonisationswelle um 1960 und mit der Entstehung vieler neuer souveräner Nationen im ehemaligen Einflussbereich der europäischen Kolonialmächte die Frage der weltwirtschaftlichen Ungleichheit die Agenda der internationalen Organisationen fast vollständig dominiert hatte. Als programmatische Grundlage der Konferenz diente das Buch Wie retten wir unsere Erde? Umweltschutz: Bilanz und Prognose von Barbara Ward und René Dubos (1972). Es liest sich als ein flammendes Plädoyer für „Die Einheit des Planeten“, so der Titel des ersten Kapitels. Es postulierte eine „Einheit der Wissenschaften“ mit Blick auf die Folgekosten der hochentwickelten Technologien, die als Hauptquelle der Umweltbedrohung identifiziert wurden. Gemeint waren z.B. die intensive, wissenschaftsbasierte Landwirtschaft oder auch die Gewinnung von Energie durch die Kernspaltung. Das Buch nahm ferner die Problematik der sogenannten ,Entwicklungsländer“ an zentraler Stelle auf und entwarf die Perspektive einer ,,planetarischen Ordnung“ der Umweltgerechtigkeit.

\footnotetext{
1 „In den verschiedenen Sprachen Gabuns gibt es kein Wort für Natur. Es scheint sogar angezeigt, die Idee von Émile Durkheim aufzunehmen, wonach Natur nicht existieren kann in Gesellschaften, die die modernen Wissenschaften nicht kennen und keinen Fundamentalgegensatz zwischen Natur und Kultur ziehen. In der Sprache Fang ist, enyeng ' das Wort, das am ehesten dem Konzept von Natur entspricht, das die modernen Gesellschaften prägt. Seine genaueste Übersetzung heißt ,Leben', aber , enyeng' verweist auch auf den Umstand der Existenz und damit auf die Welt“ (eigene Übersetzung).
} 
Damit war die Latte hoch gesetzt. Vor diesem Hintergrund ist die Berichterstattung des Korrespondenten der Neuen Zürcher Zeitung aus Stockholm zu lesen. Heribert Rausch, der später als Professor an der Juristischen Fakultät der Universität Zürich das Schweizer Umweltrecht maßgeblich prägen sollte, schrieb 1972 über die Eröffnungskonferenz:

Im Übrigen verwenden die verschiedenen Delegationen die ihnen zustehenden fünfzehn Minuten vorab dazu, die Bemühungen ihres Landes um den Umweltschutz in ein möglichst gutes Licht zu rücken. Das fällt umso leichter, als die Thematik der Konferenz nicht ausdrücklich definiert worden ist. Die offenbar in allen fünf UNO-Sprachen bestehende Verschwommenheit des Begriffs der Umwelt macht es auch möglich, dass einzelne Delegierte vorab über die Hygiene am Arbeitsplatz, andere über den Export von Rohstoffen aus Entwicklungsländern in Industrieländer, wieder andere über die drohende Ausrottung des Walfisches sprechen. ${ }^{2}$

Das Fazit des NZZ-Korrespondenten klang dann wenige Wochen später durchaus positiver. Das in Stockholm verabschiedete Schlussdokument enthielt Empfehlungen bezüglich klar definierter Themen. Es waren allerdings noch immer viele sehr unterschiedliche Aspekte, die mit der Weltumwelt in Zusammenhang gebracht wurden. $\mathrm{Zu}$ den angesprochenen Themen gehörten die möglichen Auswirkungen der Luftverschmutzung auf Wetter und Klima, der globale Energiehaushalt (fossile Brennstoffe und andere Energieträger), die Meeresverschmutzung, namentlich durch Öl und durch das Versenken von Abfällen, sowie die mittelbare Meeresverschmutzung durch die abnehmende Qualität des einfließenden Süßwassers und der Luft. Weitere Themen waren die Erhaltung bedrohter Tier- und Pflanzenarten und die Sicherung des globalen Waldbestandes. Viel Aufmerksamkeit erfuhr auch die globale Emission von Schwermetallen wie Blei, Cadmium und Quecksilber. Ein gesondertes Thema war das Insektizid Dichlordiphenyltrichlorethan (DDT), das weltweit ausgebracht worden war und sich in der Nahrungskette akkumulierte. Auch andere chlorierte Kohlenwasserstoffe sowie künstliche Düngemittel (Phosphate) in der Landwirtschaft und insbesondere die radioaktiven Abfälle beschäftigten die Delegierten. Ferner sprachen sie über Naturkatastrophen und den Lärm sowie über die Möglichkeiten der Siedlungsplanung in Umweltschutzhinsicht. Ganz zentral war schließlich die Nord-Süd-Problematik, die sich nach dem Ende der europäischen Imperien als Wohlstandsgefälle offenbarte. Man debattierte mögliche negative Einflüsse von Maßnahmen zum Schutz der Umwelt auf den internationalen Handel, insbesondere mit Rücksicht auf die wirtschaftlichen Entwicklungspotenziale armer Länder.

Alle Delegationschefs, die zum Schluss nochmals das Wort ergriffen, brachten die Überzeugung zum Ausdruck, dass die künftigen Generationen die Stock-

\footnotetext{
2 Rausch, Heribert: Ungewisse Erfolgsaussichten der Umweltkonferenz, in: NZZ, 09.06.1972, S. 1. Hervorhebungen im Original.
} 
holmer Konferenz als einen Wendepunkt in der Geschichte des Planeten Erde betrachten werden. Dies war mehr als blosse Rhetorik. ${ }^{3}$

So schloss Heribert Rausch seine Berichterstattung. Offenbar war es gelungen, aus der Vielzahl der Sachverhalte einen Ansatz für eine konzise Problemwahrnehmung zu entwickeln. Die Angleichung der wirtschaftlichen Entwicklungsmöglichkeiten von Ländern des Südens an jene des Nordens stand dabei im Vordergrund. Deshalb erhielt die aus der Stockholmer Konferenz hervorgehende neue UNO-Sonderorganisation „United Nations Environmental Program“ (UNEP) in einem Dritt-Welt-Land ihren Sitz, in der kenianischen Hauptstadt Nairobi.

Die Rede von einem „Wendepunkt“ in der materiellen Geschichte des Planeten war leider entgegen der Einschätzung des NZZ-Korrespondenten in erster Linie doch programmatische Rhetorik. Ein tatsächlicher Wendepunkt lässt sich an der Stockholmer Konferenz hingegen in Bezug auf die Wahrnehmung von weltumweltpolitischen Handlungszusammenhängen beobachten. Die mit der europäischen Expansion einher gegangene Angleichung der biologischen Umwelt hatte zu einer zunehmenden Sensibilität für Systemzusammenhänge der Weltumwelt geführt. Das bildete sich auch in der geschichtswissenschaftlichen Forschung ab.

Als der Bielefelder Geschichtsprofessor Joachim Radkau 2000 eine Weltgeschichte der Umwelt vorlegte (Radkau 2000), reagierten viele Historikerinnen und Historiker noch eher skeptisch, da sie sich im Zuge des Cultural Turn stark auf mikrohistorische Ansätze fokussiert hatten und großen, weltumspannenden Geschichtserzählungen auch vor dem Hintergrund der Postcolonial Studies eher mit Zurückhaltung begegneten. Diese standen im Verdacht, eurozentrische Meistererzählungen unkritisch zu reproduzieren. Freilich hat gerade die Umweltgeschichte relativ früh auf die Bedeutung von planetarischen Zusammenhängen hingewiesen. Dabei sticht der von Christian Pfister 1995 herausgegebene Sammelband zum 1950er-Syndrom hervor, in dem ein massiver und welthistorisch dramatischer Anstieg von umweltrelevanten Umsätzen bezüglich Energieverbrauch, Raumbedarf und Emissionen belegt wird, der ursächlich mit den Modernisierungserfolgen industrieller Volkswirtschaften ab den 1950er-Jahren verbunden sei (Pfister 1995; s. a. McNeill 2000).

Seither hat sich der geschichtswissenschaftliche Mainstream hin zur Globalgeschichte bewegt und kulturwissenschaftlich geöffnet. Es erscheinen kaum noch umwelthistorische Arbeiten, die nicht weltweite Ökosystemzusammenhänge als zumindest impliziten Untersuchungshintergrund aufspannen, neuerdings vermehrt unter dem semantisch überbordenden Stichwort „Anthropozän“ (Renn und Scherer 2015). Und vor allem hat sich die umweltgeschichtliche Forschung von der Beschreibung materieller Verhältnisse auf die Analyse der gesellschaftlichen Selbstverständigung, der Leitdiskurse und der einschlägigen (globalen) Organisationsinnovationen ausgeweitet. Exemplarisch für den boomenden Forschungsstand, der hier nicht gesamthaft referiert werden kann, sind die Arbeiten von Perrin Selcer, der die Bedeutung der UNO als Vermittlungsinstanz zwischen der wissenschaftlichen Umweltexpertise und den weltpolitischen Gremien mit Blick auf die Entstehung eines politischen Gegen-

\footnotetext{
${ }^{3}$ Rausch, Heribert: Positive Bilanz der Stockholmer Umweltkonferenz, in: NZZ 19.06.1972, S. 3. Hervorhebungen im Original.
} 
stands Umwelt rekonstruiert hat (Selcer 2018). Verwiesen sei auch auf den Innsbrucker Umwelthistoriker Patrick Kupper, der in kritischer Auseinandersetzung mit dem von Pfister geprägten Begriff des „1950er-Syndroms“ eine „1970er-Diagnose“ postulierte. Erst mit einer deutlichen zeitlichen Verzögerung gegenüber dem dramatischen Anstieg umweltrelevanter Stoffumsätze sei dieser Vorgang gesellschaftlich wahrgenommen worden (Kupper 2003). Dies ging mit einer radikalen Neufassung des Begriffs „Environment“ - Umwelt - einher (Warde et al. 2019). Joachim Radkau hat in diesem Zusammenhang von einer „Ära der Ökologie“ gesprochen (Radkau 2011), die in den Jahren um 1970 begonnen habe.

Dieser Wahrnehmungswandel ist Teil eines Einschnitts, der weit über die Sachproblematik der Weltumwelt hinausweist (Hünemörder 2005; Kuchenbuch 2012). Er hat mit zwei technowissenschaftlich angetriebenen Erkenntnisprozessen zu tun. Der eine war ein eher unerwarteter Nebeneffekt der US-amerikanischen und der sowjetischen Weltraumprogramme, die seit den 1950er-Jahren mit viel Geld betrieben wurden. Das weltgeschichtlich bedeutsamste Ergebnis des Kalten Kriegs ist vermutlich das Bild der Erde aus dem Weltall, das die Besatzung der Apollo-17Mission 1972 machte. „Blue Marble“ - die Erde als verletzliches geschlossenes System angesichts der weiten Leere rundherum.

Der andere Prozess verweist auf die Geschichte der Computer. Mit der rasanten Automatisierung der Datenverarbeitung durch große Rechenmaschinen eröffnete sich ab den späten 1950er-Jahren die technische Möglichkeit kybernetischer Systemmodellierungen (Campbell-Kelly et al. 2016; Seibel 2016). Fachleute des Operations Research wie Jay Forrester machten sich daran, industrielle Fabrikationsprozesse systemisch zu modellieren und übertrugen diese Methodik bald auf soziale Kollektive wie Städte, Volkswirtschaften und schließlich die ganze Welt. Wie eine Bombe schlug 1972 der Bericht des Club of Rome zu den Grenzen des Wachstums weltweit ein, der basierend auf Forresters Systematik und unter Einsatz eines der damals leistungsfähigsten Mainframe-Computer am MIT in Boston aus den Kenngrößen der Weltbevölkerung, des globalen Ressourcenangebots und der Umweltschädigung durch moderne Produktionspraktiken die dramatische Zukunftsvision eines globalen Kollapses errechnete.

Passend zum Bild der „Blue Marble“ machte das ihm zugrunde liegende Flussdiagramm die Möglichkeit der Weltberechnung plausibel - als wäre die Erde ein Raumschiff, dessen Flugbahn in die Zukunft sich von einem computertechnisch hochgerüsteten Kontrollzentrum aus für alle Erdbewohnerinnen und -bewohner in einer optimalen Weise steuern ließe (Höhler 2015). Naturwissenschaftlich fundierte Modellierungen eines Weltsystems gehören heute $\mathrm{zu}$ den wichtigen kognitiven Ressourcen der Weltumweltpolitik (Rahmstorf und Schellnhuber 2019). Vor diesem historischen Hintergrund ist der hier versuchsweise eingeführte Begriff einer Weltumwelt zu lesen. Aus systemtheoretischer Perspektive wundert es nicht, dass soziale Systeme ein Außenverhältnis zu bestimmen haben, das mitunter sehr konkret die Umwelt als Natur meint(e). Zu fragen ist dann, wie es dazu kam, dass sich trotz der Heterogenität der Naturphänomene und der Vielfalt ihrer Beobachtungsweisen ein numerisches Deutungsmodell durchsetzen konnte, das global konsensfähig und weltweit kommunizierbar ist. 
Um die hier interessierenden Aspekte der Ökonomisierung und der Quantifizierung genauer zu verstehen, ist ein Periodisierungsvorschlag passend, den die Historikerinnen Lea Haller, Sabine Höhler und Andrea Westermann 2014 gemacht haben. Mit Blick auf das ökonomische Kalkül in der Naturwahrnehmung schlagen sie drei historische Phasen vor. Erstens habe es eine Zeit der kameralistischen Naturerfassung gegeben, die von der biologischen Systematik Carl von Linnés her den Diskurs des 18. und noch des 19. Jahrhunderts geprägt habe. Zweitens beobachten sie eine Zeit der geopolitisch geprägten Weltumweltwahrnehmung von ca. 1900-1970, in der im Zeichen der kapitalistischen Expansion Europas der Fokus ausschließlich auf die Nutzbarmachung von Bodenschätzen gerichtet gewesen sei. Und drittens sprechen sie von einem Zeitalter der umweltökonomischen Natur seit etwa 1960 (Haller et al. 2014).

Damit ist ein hilfreicher chronologischer Rahmen gesetzt. Der Bruch der 1970erJahre betrifft Organisationsinnovationen auf den Ebenen der nationalen Staatlichkeit und der internationalen Organisation und dazu auch neue und sich zunehmend global vernetzende soziale Bewegungen. Es geht ferner um die neuen technischen Möglichkeiten der Erdsicht aus dem All und um die Proliferation von Wahrnehmungsweisen des Sozialen in der analytischen Sprache der Wirtschaftswissenschaften. In allen diesen Hinsichten scheint den Praktiken der Quantifizierung seit 1970 eine besondere Bedeutung zuzukommen.

\section{Die Bedeutung von Quantifizierung für die globale Kommunizierbarkeit von Umwelt}

Inwieweit die Umweltkonferenz von Stockholm 1972 für die Erhaltung des planetarischen Lebensraums selbst einen Wendepunkt darstellt, ist offen. Sie war es auf jeden Fall in wahrnehmungsbezogener und vor allem auch in organisatorischer Hinsicht.

In der Geschichte des UN-Systems stellte die Konferenz nach der Welthandelsund Entwicklungskonferenz (UNCTAD), die 1964 in Genf stattfand, und nach der UN-Menschenrechtskonferenz von 1968 erst den dritten thematisch fokussierten Großanlass dar. Bis dahin waren weltpolitische Gegenstände in der Regel auf den Sitzungen der Generalversammlung verhandelt worden, wo sich exklusiv souveränitätstheoretisch legitimierte Länderdelegationen trafen. In Stockholm kamen hingegen ganz unterschiedliche Akteure zusammen, so wie dies in den folgenden Jahren bei den thematischen Großkonferenzen üblich werden sollte. Auch auf der ersten Weltfrauenkonferenz in Mexiko 1975 und den später stattfindenden Umweltkonferenzen, allen voran in Rio 1992, versammelte die UNO nicht nur Vertreterinnen und Vertreter von souveränen Staaten, sondern auch Wissenschaftlerinnen und Wissenschaftler sowie Delegationen der nun immer zahlreicher werdenden weltweit tätigen Nichtregierungsorganisationen. Überdies entsandten verschiedene Länder neben den diplomatischen Abordnungen hochrangige Beamte aus dem innenpolitischen Verwaltungsapparat. Ferner waren Dachorganisationen von Umweltverbänden präsent; so namentlich die International Union for the Conservation of Nature (IUCN), die nach dem Zweiten Weltkrieg als Verbund von nationalen Natur- und Heimat- 
schutzorganisationen sowie wissenschaftlichen Körperschaften gegründet worden war (Schleper 2019). Und auch eine ganze Reihe von internationalen Organisationen außerhalb des UN-Systems, wie die OECD oder die EWG, schickten Beobachter (McCormick 1989, S. 88-105).

Wegen der von Heribert Rausch beobachteten „Verschwommenheit des Begriffs der Umwelt" zeigen sich historisch unterschiedliche Pfade der Art und Weise, wie Umwelt wahrgenommen und politisch darauf reagiert wurde. Ihr Zusammenlaufen in Stockholm bedeutete, dass man nicht einfach auf diplomatische Routinen und Protokolle zurückgreifen konnte, sondern ganz unterschiedlichen Kommunikationskulturen gerecht werden musste, die je verschieden mit sozialen Hierarchien umgingen. Auch in dieser Hinsicht war die Praktik der Quantifizierung kommunikativ hilfreich, da Zahlen Objektivität insinuieren.

Der Weltgesellschaftssoziologe John Meyer hat mit seinen Mitarbeitenden die Entstehung eines globalen Umweltschutzregimes empirisch zur Kritik am realistischen Ansatz in der Analyse der internationalen Beziehungen verwendet. Im Gegensatz zur realistischen Auffassung, dass die Nationalstaaten die entscheidenden Akteure sind und das globale Umweltschutzregime das Produkt ihrer Interessenpolitik ist, zeigen Meyer et al. (2005), dass sich das globale Umweltregime jenseits der Nationalstaaten und teilweise auch an ihnen vorbei herausgebildet hat. Die entscheidenden Impulse seien nicht von den Nationalstaaten ausgegangen, sondern von der Wissenschaft und von privaten Organisationen. Dabei habe die Rationalisierung des Umweltverhältnisses durch die moderne Wissenschaft eine zentrale Rolle gespielt. Dieser Punkt stimmt ungefähr mit dem überein, was hier unter Quantifizierung verstanden wird. Alle genannten Faktoren sind eng verknüpft. Denn erst mit der Verwissenschaftlichung der Natur, ihrer Klassifikation, Vermessung und Modellierung, konnten sich Umweltschutzverbände auf wissenschaftliche Erkenntnisse berufen, um ihre Forderungen gegenüber nationalstaatlichen Autoritäten zu legitimieren.

Entlang der von Haller et al. (2014) vorgeschlagenen Periodisierung lassen sich die Genealogien dieser verschiedenen Akteure historisch ordnen, wobei Privatpersonen und private Vereine eine wichtige Rolle gespielt haben. Dabei ist zu fragen, wann und wie sie Quantifizierungspraktiken verfolgt oder auch erfunden haben. Als grobes inhaltliches Raster ist es zudem hilfreich, die inhaltlich überbordende Umweltproblematik gemäß einem Vorschlag von John McNeill nach den Sphären des Stoffwechsels zu gliedern. Gemeint sind die Aquasphäre, die Geosphäre, die Biosphäre und die Atmosphäre (McNeill 2000). Man ist mit einer komplexen, interagierenden Konstellation von Akteuren konfrontiert, die Privatpersonen, lokale bürgerliche Vereine und zunehmend auch nationalstaatliche Institutionen umfasst, wobei sich die interessierten Individuen und die nichtstaatlichen Vereine schon relativ früh geografisch weit zu vernetzen begannen und später auch die umweltpolitisch aktiven Staatsorgane international zu kommunizieren begannen.

Im Zuge der Entstehung der modernen Naturwissenschaften um etwa 1700 stand zunächst die Biosphäre im Vordergrund. Namentlich durch den Schweden Carl von Linné ist die botanische und die zoologische Systematik zu einem Rückgrat der modernen Umweltwahrnehmung geworden. Sie erlaubte es, die Vielfalt der Erscheinungen des Lebens zu kategorisieren, quantitativ zu erfassen und vielfältige Vergleiche anzustellen. 
Für die Biologie wird mittlerweile mit Blick auf die Bedeutung dieser Praktiken sogar argumentiert, sie sei überhaupt als eine frühe Form der Datenwissenschaft entstanden. Im Gegensatz etwa zur Medizin, der anderen Linie innerhalb der LifeSciences, habe sie sich nicht als Experimentalwissenschaft konstituiert, sondern ihre Wissenschaftlichkeit durch vielfältige, systematische Quantifizierungspraktiken gewonnen (Strasser 2012). Bei den Taxonomien des 18. Jahrhunderts (und vermutlich auch noch des frühen 19. Jahrhunderts) ging es allerdings noch primär um das Sammeln, Beschreiben und Zeichnen von Gefundenem im Sinne einer qualitativen Feldforschung. Deren Übergang zu Quantifizierungspraktiken fand erst später statt, die qualitativen Verfahren legten hierfür aber wesentliche Grundlagen. Ohne sie wären nicht nur das Konzept von Biodiversität, sondern auch die heutige Vorstellung eines wissenschaftlich bearbeitbaren Gegenstands „Biologie“ nicht möglich gewesen. Die frühe Quantifizierung des Klimas hat andere Paten. Aber auch in diesem Zusammenhang stand am Anfang der Quantifizierung das mehr qualitativ motivierte Staunen über die Vielfalt natürlicher Phänomene.

Die treibenden Kräfte waren bis erstaunlich weit ins 20. Jahrhundert hinein Privatpersonen, deren Namen in der Benennung vieler Vorkommnisse und Organismen bis heute dokumentiert sind. Zum einen handelte es sich um Gelehrte, die mit beachtlicher Akribie Kategorien der Lebensbeschreibung erfanden und inhaltlich füllten. Zum anderen formierte sich rund um die biologische Systematik auch eine bürgerliche Selbstpraktik. Wie Sophie Ruppel gezeigt hat, wurde das Anlegen einer persönlichen Sammlung von selbst beobachteten Arten um 1800 gerade auch für bürgerliche Frauen zu einem wichtigen Medium der Identitätskonstruktion (Ruppel 2019). Beim Fangen von Schmetterlingen und beim Anlegen eines Herbariums wurde Identität eingeübt und zugleich Umwelt quantifiziert. Dabei dienten überragende Naturforscher wie Alexander von Humboldt als Vorbilder, die alles sammelten und kategorisierten, was ihnen die Natur auf ihren Weltreisen darbot (Daum 2019).

Das Auszählen von Naturphänomenen als bürgerliche Selbstpraktik griff im 19. Jahrhundert von der Biosphäre auch auf die Geosphäre, die Aquasphäre und die Atmosphäre aus. Das tägliche Notieren von Regenmengen und von lokalen Temperaturen wurde z. B. in der Schweiz zu dieser Zeit ebenfalls zu einer bürgerlichen Selbstdisziplinierungsform. Aus der Summe aller Einzelbeobachtungen resultierte ein national generalisiertes Umweltwissen (Hupfer 2019), das anschlussfähig an globale Deutungssysteme wurde. Nichtstaatliche naturforschende Gesellschaften boten beim systematischen Abgleich dieser Praktiken einen entscheidenden institutionellen Rahmen (Bürgi 2004). Frühe Ansätze zu einer weltumweltpolitischen Konzeption in Zahlenform finden sich ferner in den vielen statistischen Kommissionen der zweiten Hälfte des 19. Jahrhunderts. Wissenschaftler (fast ausschließlich Männer) trafen sich z. B. im Jahr 1883 in Washington auf der internationalen Meridiankonferenz, bei der ein geodätisches Vermessungsnetz über den ganzen Planeten gelegt wurde. Die Vereinheitlichung der Zeit war damals ebenfalls ein Thema (Ogle 2015).

Mit Blick auf die Institutionalisierung von Akteuren der quantifizierenden Umweltbeobachtung ist der Übergang von der ersten Phase in der von Haller et al. (2014) vorgeschlagenen Periodisierung, also der Zeit der ,,kameralistischen Naturerfassung“, zur zweiten Phase, der ,Zeit der geopolitisch geprägten Weltumweltwahr- 
nehmung“, die sie auf die Jahre von etwa 1900-1970 datieren, eher unscharf. Zwar bildete die Optimierung von nationalstaatlicher Herrschaft in der Summe der bürgerlich-individuellen Quantifizierungspraktiken den konzeptionellen Fluchtpunkt. Aber die entscheidenden Akteure blieben noch lange Privatpersonen, die sich eine auf wissenschaftlich quantifizierter Naturwahrnehmung basierende, rationale Ausübung von Herrschaft erträumten und deren Möglichkeit befördern wollten, die aber fast nie - und eigentlich erst im 20. Jahrhundert - tatsächlich im Auftrag von Staaten handelten. Das gilt für die soziale Relevanz aller Vorgänge des Stoffwechsels in der Luft, in der Erde, im Wasser und im Bereich der lebenden Organismen; und es trifft für alle Beobachtungsformen zu, sei es Kartografie oder Statistik.

Neuere Arbeiten zur politischen Geschichte des Zählens seit der Aufklärung betonen die Unvollständigkeit und die Kontingenz in dem Versuch sich modernisierender Staatswesen, Herrschaftsentscheide auf Zahlen zu gründen und zu ihrer Generierung nationalstaatliche Statistikämter zu institutionalisieren (Behrisch 2016). Das betrifft auch, wie Peter Kramper (2019) nachgewiesen hat, die ganz grundsätzliche Problematik der Vereinheitlichung der Nomenklatur im Messwesen, die noch bis ins 20. Jahrhundert hinein in der Alltagspraxis erstaunlich vielfältig und lokal geblieben ist.

Es geht um den Nationalismus oder um die Ausrichtung des umweltpolitischen Handelns an der Vorstellung einer Konkurrenz der Nationen, deren Ansprüche auf den Zugriff zu den Ressourcen der Umwelt im immer klarer werdenden Horizont ihrer Beschränktheit zu diplomatisch komplexen Kommunikationen und zu vielen zwischenstaatlichen Kriegen geführt haben. An der Epochenschwelle um 1900 entstand in den sich modernisierenden Gesellschaften ein neues Verhältnis zur Natur, das chauvinistisch funktionierte und zum Ausflaggen der eigenen Besonderheit ganz wesentlich das Umweltverhältnis bediente oder nutzte. Die Optimierung einzelner, quantitativ abgrenzbarer Volkswirtschaften gegeneinander hat die Verwandlung der Weltumwelt in ein Gefüge von Zahlen zu Beginn des 20. Jahrhunderts wesentlich befördert. Die bürgerliche Selbstpraktik der numerischen Naturbeobachtung trug im Staatsbildungsprozess gerade mit dem ihr inhärenten Zug zur nichtstaatlichen Vereinsbildung wesentlich zur Konsolidierung und Plausibilisierung der nationalstaatlichen Weltordnung bei. Im Medium der Naturbeobachtung konkretisierte sich so eine spezifische Form des ,banalen Nationalismus“ (Billig 1995). So wurde z.B. das Edelweiß zu einer Schweizer Nationalblume.

In der Organisationsgeschichte der Umweltfrage gilt die Gründung des ,Sierra Club“ im Kontext der Schließung der „Frontier“ in den Vereinigten Staaten im ausgehenden 19. Jahrhundert als impulsgebende Innovation. Sein Hauptanliegen war das Ausscheiden von räumlich definierten Reservaten als Nationalparke, in denen der infrastrukturelle Modernisierungszugriff verboten wurde. Das machte schnell auch in anderen sich industrialisierenden Ländern wie der Schweiz Schule, wo zu Beginn des 20. Jahrhunderts ein alpiner Nationalpark definiert wurde (Kupper 2012). Vielerorts entstanden damals privatrechtliche Vereine zum Schutz der Natur, heute würde man von Nichtregierungsorganisationen (NGO) sprechen.

Während die Parke mit dem Adjektiv „national“ versehen wurden, trugen auch die entsprechenden Organisationen fast überall einen expliziten Lokalbezug mit. Die gesteigerte Aufmerksamkeit und Sorge für den Schutz begrenzter Lebensräu- 
me für klar abgezirkelte Bevölkerungen vor dem Hintergrund der kapitalistischen Expansion und des Nationalismus äußerte sich besonders deutlich in der Umwelt-, Lebensraum-, und Naturpolitik des nationalsozialistischen Regimes in Deutschland zwischen 1933 und 1945 (Radkau und Uekötter 2003). Tatsächlich handelte es sich hierbei aber trotz der übersteigerten national-chauvinistischen Rhetorik um eine globale Angelegenheit. Dies erstens in organisatorischer Hinsicht, indem sich die neuen nationalen Naturschutzakteure global verglichen und im Austausch gegeneinander abgrenzten (Gissibl et al. 2015). Und zweitens inhaltlich, was beim Vogelschutz, einem frühen Thema der umweltpolitischen Kommunikation, besonders deutlich wird. Weitere nichtstaatliche Akteure im Bereich der Umweltpolitik entstanden in den 1960er-Jahren. Vermutlich als Reaktion auf die von Christian Pfister beobachtete Verschärfung der Umweltwirkung von sich modernisierenden Gesellschaften ab den 1950er-Jahren geriet zunehmend die ganze Welt in den Blick. 1961 wurde im Westschweizer Städtchen Gland der World Wildlife Fund (WWF) gegründet (Schwarzenbach 2011). Mit den sozialen Bewegungen rund um das Wunderjahr 1968 entstanden weitere global wirksame Nichtregierungsorganisationen wie Greenpeace oder Friends of the Earth (Radkau 2011).

Zeitgleich mit der Entstehung nationalistischer, aber privat organisierter Umweltverbände machten sich auch die Staatsverwaltungen an die Quantifizierung der Natur, zunächst namentlich mit Blick auf die Geosphäre mit dem Aufbau systematischer Arealstatistiken (Schneider 2004). Und mit Blick auf die Schifffahrt auf den Weltmeeren begannen auch die imperialen Mächte um 1900, die Gezeiten präzise zu erfassen. Sie beschäftigten schon früh im 20. Jahrhundert ganze Heerscharen von Rechnerinnen, die in den statistischen Büros, z. B. der britischen, US-amerikanischen und sowjetischen Marine, als menschliche Computer nach entsprechenden Vorgaben auf Tage, Monate und Jahre hinaus für alle Häfen der Welt den Wasserstand berechneten (Campbell-Kelly et al. 2016, S. 46-50). Das war eine wichtige Vorfront der Datafizierung globaler Umweltverhältnisse, die zugleich zur Frühgeschichte des Computers gehört.

Viele heute als Beispiele von „Weltumwelt“ geltende Sachverhalte wurden im 20. Jahrhundert zu Staatsaufgaben. Mit der zunehmenden Macht der nationalstaatlichen Ordnung und der wachsenden Plausibilität des quantifizierenden Zugriffs auf die Natur entstand z.B. das neue Politikfeld der Raumplanung. Es ging einher mit der Vorstellung, quantitativ erfasste Naturstücke könnten in ein numerisches Äquivalenzverhältnis gebracht und abgetauscht werden. Ähnliche Quantifizierungsphänomene sind auch im Management der Aquasphäre zu verzeichnen. In der Schweiz führten zahlenförmige Grenzwerte der Wasserqualität ab den 1950er-Jahren zu einem gigantischen Staatsprojekt. Flächendeckend wird seither das Siedlungsabwasser in Reinigungsanstalten kanalisiert (Gugerli 2000).

Aber erst um 1970 bildeten die modernen Staatsverwaltungen eigene Behörden für die Umweltfrage aus. Den Anfang machte Schweden 1967, gefolgt von den USA 1970 und Japan 1971. Die Gründung eines staatlichen Umweltministeriums folgte in Australien, Österreich, Kanada, Dänemark, Ostdeutschland, Frankreich, Italien, Norwegen und im Vereinigten Königreich bis 1973. Vermutlich in Reaktion auf die Konferenz von Stockholm lässt sich zehn Jahre später eine zweite Welle der Ausdifferenzierung nationaler Bürokratien beobachten. Sie wurde von Brasilien angeführt 
(1982), gefolgt von Finnland, den Niederlanden, der Schweiz und Westdeutschland (Kaiser und Meyer 2017, S. 3). Die Weltgesellschaftssoziologin Anne Hironaka hat diesen Homogenisierungsprozess detailliert beschrieben und gezeigt, wie auf die Welle der Amtsgründungen eine entsprechende Welle der Gesetzgebungen und Verordnungen folgte, die sich in allen Ländern an ähnlichen Kommunikationsformen und Normen vornehmlich quantitativer Art orientierte (Hironaka 2014). Es ist insofern der Beobachtung von Meyer et al. (2005) zuzustimmen, dass die Nationalstaaten an der Entstehung eines globalen Umweltschutzregimes nur marginal beteiligt waren. Es waren vielmehr private und wissenschaftliche Akteure, die den umweltpolitischen Diskursraum absteckten, in den sich die Staaten erst sehr spät einklinkten.

\section{Der Zusammenhang von Quantifizierung und ökonomischen Denkmodellen in der Umweltpolitik}

Als neueste Phase haben Haller, Höhler und Westermann in ihrem Periodisierungsschema ein Zeitalter der umweltökonomischen Natur vorgeschlagen, das in den 1960er-Jahren einsetzte (Haller et al. 2014). Mit Blick auf die durch Quantifizierungspraktiken neuerdings plausibel scheinende Wahrnehmung von Umwelt als einer volkswirtschaftlichen Größe ist dieser Diagnose sicher zuzustimmen (Lucht und Spangardt 2005). Gleichwohl stellen sich einige Fragen.

Die Feststellung einer Ökonomisierung der Weltumwelt im ausgehenden 20. Jahrhundert steht im Kontext der Kritik am Neoliberalismus. Die gängige Deutung behauptet, dass in der gesellschaftlichen Selbstverständigung und motiviert durch die Weltwirtschaftskrise der 1970er-Jahren Deregulierungspraktiken die ältere Vorstellung, gesellschaftliche Probleme ließen sich durch staatliche Vorschriften, Verbote und Gebote lösen, an den Rand gedrängt hätten. Stattdessen sei die Wirtschaftswissenschaft zur wichtigsten sozialwissenschaftlichen Beratungsagentur der Politik aufgestiegen, die als zentrale Handlungsanweisung das Setzen von individuellen Anreizen definiere (Harvey 2005; Ptak 2008). Im Zuge der Quantifizierung von Natur hat eine ursprünglich eher periphere Forschungsarbeit des US-amerikanischen Ökonomen Ronald Coase von 1960 gewaltige Resonanz erlangt. Er schlug damals vor, externe Wirkungen als ,soziale Kosten“ in das ökonomische Kalkül aufzunehmen und auch umweltbezogene Handlungen so zu modellieren (Coase 1960). In der dominanten neoliberalismuskritischen Forschung wird dies als eine kapitalistische Enthemmung dargestellt, die das egoistische Gewinnstreben seit den 1970er-Jahren ungehindert auch auf Gemeingüter wie die natürlichen Ressourcen ausgreifen lasse.

Im Licht der oben ausgeführten „Verschwommenheit des Begriffs der Umwelt“ ist jedoch anzunehmen, dass der Zusammenhang zwischen weltumweltpolitischer Kommunikation, den Prozessen der Quantifizierung und jenen der Ökonomisierung deutlich komplexer und historisch älter ist. Wie Rüdiger Graf gezeigt hat, standen sich in der Umweltpolitik sowohl national als auch international nach dem Zweiten Weltkrieg zwei Handlungsoptionen entgegen: Auf der einen Seite die obrigkeitliche Intervention, z. B. durch das Festlegen von naturwissenschaftlich (also quantitativ) definierten Grenzwerten bei der Umweltbelastung in allen Sphären; und anderer- 
seits das Öffnen von individuellen Handlungsanreizen vor dem Hintergrund einer wiederum quantitativ begründeten Naturwahrnehmung (Graf 2019). Anstatt von dem Gespenst einer neoliberalen Weltherrschaft im diffusen Begriffsfeld von Quantifizierung und Ökonomisierung auszugehen, scheint es interessanter, die gesellschaftliche Bedeutung von Buchhaltungspraktiken genauer in den Blick zu nehmen.

Die Quantifizierung der Weltumwelt wird heute in erster Linie als eine Ökonomisierung von Natur diskutiert (und betrieben). Dabei wuchs die Erkenntnispraktik der Buchhaltung zu zentraler Bedeutung auf (Hopwood 2009). Ein Grund dafür ist ihre Nähe zur Handlungsfähigkeit von Gesellschaften. Die Buchhaltung ermöglicht es, die ganze Welt mit ihrem Naturbezug im Modell einer Firma zu imaginieren, sodass Kosten und Nutzen fein säuberlich getrennt werden können und sich Verantwortlichkeiten klar zuschreiben lassen. Freilich ist die Geschichte der Buchhaltung anders getaktet als die Geschichte der neoliberalen Ideologie und beide unterscheiden sich bezüglich ihrer zeitlichen Entfaltung von der globalen Umweltwahrnehmung. Die Geschichte der Buchhaltung zeigt historisch eine gewaltige Plausibilitätszunahme, und zwar weltweit seit dem 17. Jahrhundert. Dabei ist in erster Linie an die Kommodifizierung des Grundeigentums zu denken. Ohne die katastergeometrische Vermessung von einzelnen Parzellen und der numerischen Beobachtung von deren Erträgen wäre wohl überhaupt die Genese des modernen Kapitalismus nicht denkbar gewesen (Macpherson 1962). Das war bereits ökonomische Quantifizierung von Natur in Reinkultur - vor rund 350 Jahren.

Die kulturgeschichtliche Tiefe des hier interessierenden Zusammenhangs zeigt sich exemplarisch am Begriff der „Nachhaltigkeit“. Er bezog sich anfänglich im 18. Jahrhundert eng auf die Bewirtschaftung von Waldflächen. Gemeint war das Zählen der Holzmenge in einem definierten Waldstück und dessen Korrelation mit dem ebenfalls numerisch gefassten Wachstumspotenzial der Bäume zwecks der Errechnung einer maximalen Erntemenge, die den Bestand nicht reduziert, sodass die Holzressourcen auch künftigen Generationen noch zur Verfügung stehen. Holz war bis zur Entdeckung und Nutzbarmachung der fossilen Kohlenstoffvorräte die wichtigste Energieressource im globalen Industrialisierungsprozess. Entsprechend reich ist die Geschichte ihrer Quantifizierung. In den USA beispielsweise, deren Wirtschaft bis überraschend weit ins 20. Jahrhundert hinein auf Holz als Energiequelle und als Baustoff basierte, war die genaue quantitative Erfassung der vorhandenen Bestände und ihres nachhaltigen Nutzungspotenzials ein wichtiges Thema (Demeritt 2001). Auch in der Weltwirtschaft spielte das Auszählen von Holzvorräten und die damit verbundene Bestimmung des Holzpreises noch um 1900 eine zentrale Rolle (Bemmann 2019).

In der Geschichte der Umweltquantifizierung ist der Wald überdies interessant, weil in diesem Zusammenhang schon früh nicht nur geosphärische Flächen vermessen wurden, sondern hier auch die Anfänge der Quantifizierung von Biomasse liegen, die im 20. Jahrhundert zunehmend zur numerischen Vermessung der Biodiversität führten und zum Versuch, $\mathrm{CO}_{2}$-Senken zu definieren. In einem viel zitierten Aufsatz hat Morgan Robertson (2006) ethnografisch rekonstruiert, wie Umweltbehörden in den USA die Vielfalt der Bodenbedeckung quantifizierten und welche epistemischen Probleme damit verbunden waren. Die Quantifizierung von Umweltverhältnissen hat die Möglichkeit geschaffen, einzelne Territorien mit der auf ihnen 
wachsenden Biosphäre in einen gegenseitigen Verrechnungsmodus zu stellen. Haller et al. (2014) haben in diesem Zusammenhang zu Recht auf die Bedeutung von „Flächeneingriffs- und Ausgleichsregelungen in der Stadt- und Landschaftsplanung“ und auf Maßnahmen zur Kompensation der Bodenversiegelung hingewiesen (Haller et al. 2014, S. 14). Luftqualitätskompensationen wären ein anderes Beispiel.

Im Weltmaßstab sind solche Betrachtungen stark durch die Weltgesundheitsorganisation (WHO) befördert worden. Sie gab um die Jahrtausendwende eine umfassende Studie in Auftrag, die 2005 als „Millennium Ecosystem Assessment“ in Genf erschien (WHO 2005). Die darin enthaltene Quantifizierung der Weltumwelt wird seither viel zitiert und stellt eine wichtige Basis für vielerlei Weltbuchhaltungspraktiken dar (Ehlers 2008, Abb. 19). Diesbezügliche Buchhaltungen sind so omnipräsent, dass sie bereits zum Gegenstand von Kunstprojekten wurden. So konzipierte die australische Künstlerin Debbie Symons 2009 eine 5-minütige Videoinstallation mit dem Titel ,World Species Market“, die basierend auf den Angaben der International Union for the Conservation of Nature (IUCN) den biodiversen Weltzustand in Echtzeit in eine Analogie zur Visualisierung von Aktienwerten auf den Bildschirmen der Börsenhändler stellte (Höhler 2014). Diese Reflexion trägt der Präsenz und der Selbstverständlichkeit von vielfältigen numerischen Operationen im globalen Umweltverhältnis Rechnung, zu denen etwa das Ranking von BiodiversitätsHotspots gehört oder das Aufstellen von globalen Bilanzen, etwa im Konzept des „ökologischen Fußabdrucks“, oder auch die Verrechnung von $\mathrm{CO}_{2}$-Emissionen.

Bezüglich der Atmosphäre und der Klimafrage sind Quantifizierungs-, Ökonomisierungs- und Buchhaltungspraktiken seit den späten 1980er-Jahren zu größter Bedeutung aufgestiegen. Das Zählen von $\mathrm{CO}_{2}$-Partikeln pro Volumeneinheit der Luft fand aus den engen akademischen Zusammenhängen der Physik und der Chemie Eingang in die breite Öffentlichkeit. Die politische Ökonomie der diesbezüglichen Zählweisen hat der soziologischen Gesellschaftsbeschreibung seither einiges Kopfzerbrechen beschert (Callon 2009; MacKenzie 2009). Heute gibt es weltweit mehrere $\mathrm{CO}_{2}$-Emissions-Handelssysteme, deren Wechselkurs zueinander unklar ist. Sie belegen aber alle die erstaunliche Tatsache, dass die Weltpolitik die globale Umweltproblematik fast ganz über numerische Kommunikationen zu lösen können glaubt.

Die bis in die 1980er-Jahre umweltpolitisch äußerst innovativen USA übersetzten diesen Ansatz in ein Messsystem wirtschaftlicher Folgekosten bezüglich der Luftverschmutzung. Wenn eine Firma, so der Grundgedanke, durch Schwefelemissionen zur Versäuerung des Regens beitrage, müsse ihre Wirkung in Dollar und Cent ausgewiesen und in ihre Buchhaltung eingeschoben werden. Weil man Ausstöße von Industrieanlagen für das Gemeinwohl schlecht fand, sollte ihre Menge reduziert werden. Hierzu entstanden in den 1990er-Jahren erste Handelssysteme von Emissionen, die als Schulden am Gemeinwohl in konkret bezifferbar scheinenden Papieren handelbar wurden, die so einen Wert erhielten. Sie wurden mit gemeinwohlfördernden Maßnahmen bezüglich der Qualität von „Umwelt“ in anderen Sachzusammenhängen verrechenbar gemacht (Meckling 2011). In Australien wurde diese umweltpolitische Strategie bald übernommen und auf den klimawirksamen Ausstoß von $\mathrm{CO}_{2}$ übertragen (Kuch 2015). Auf der Umweltkonferenz von Kyoto 1997 stieß die Idee, Umweltschäden zu zählen und als Kosten in die Buchhaltung von Firmen und Staa- 
ten zu schreiben, auf größtes Interesse. Für die Umsetzung des Konzepts wurden verschiedene Mechanismen erfunden. Die Europäische Union führte das neue Vorgehen rasch ein, allerdings zunächst nur mit mäßigem Erfolg. Die Details dieser Geschichte sind schon oft erzählt worden (so etwa in Fatheuer et al. 2015). Sie handelt von der krassen Reduktion sehr komplexer Zusammenhänge auf ein einzelnes $\mathrm{Maß}$, nämlich $\mathrm{CO}_{2}$-Anteile pro Volumeneinheit der Luft (Moreno et al. 2015).

Im Schnittfeld der Quantifizierung und Ökonomisierung der Weltumwelt hat die Kommunikationsform des Vergleichs eine zentrale Bedeutung erlangt (s. hierzu die Beiträge in Steinmetz 2019). In den verschiedenen Emissionshandelssystemen, die sich in den letzten Jahren ausgebreitet haben, werden völlig verschiedene Umweltwirkungen miteinander in eine vergleichende Abrechnung gebracht: Die Ausstöße eines Industrieunternehmens in Deutschland erscheinen gleichwertig zum Verzicht einer Dorfgemeinschaft im Norden von Madagaskar, ein Stück Wald abzuholzen. Dabei sind die politischen Realitäten in Madagaskar und in Deutschland völlig verschieden und es bedarf kulturhistorisch weit ausgreifender Konvergenzprozesse, damit ein solcher Vergleich überhaupt Sinn macht. Zahlen spielten dabei die zentrale Rolle. Nie zuvor in der Weltgeschichte hat es so hoch abstrakte und trotzdem handlungsrelevante Kommunikationsweisen gegeben. Mit großer Selbstverständlichkeit spricht die Weltumweltdiplomatie heute von $\mathrm{CO}_{2}$ - ̈̈quivalenten. Vom methangesättigten Furz einer Kuh auf einer Schweizer Alp bis zur Verbrennungslast auf den Abfallhalden in Agbogbloshie in Ghana werden alle klimawirksamen Ereignisse auf $\mathrm{CO}_{2}$-Emissionen umgerechnet.

Bemerkenswert erscheint die politische Unentschiedenheit dieser neuartigen Buchhaltungspraxis. Es wäre sicher verkürzt, die Verwandlung der Natur in ökonomisch verwertbare Assets als Einsatz einer neokonservativen Weltverschwörung zu deuten. Es geht vielmehr um eine politisch generalisierte Kommunikationsform. Denn auch die eher linksstehenden Umweltparteien haben die Quantifizierungstechniken seit den 1990er-Jahren mit großem Elan aufgenommen. Eine Zeit lang galt es gerade für die grüne Politik als hilfreiche Strategie, im Anschluss an die bestens bewährte Quantifizierungspraktik der volkswirtschaftlichen Gesamtrechnung die in ihr versteckten Umweltwirkungen ökonomischen Handelns durch eine verfeinerte Buchhaltung sichtbar zu machen. Haller, Höhler und Westermann hielten in dem Zusammenhang fest:

Suffizienz- und Effizienzziele lagen gleichwohl eng beieinander. Beide Ausrichtungen der Ökonomik erfassten Umwelt mit Instrumenten der mathematischen Kalkulation und des ökonomischen Kalküls, beide verbanden numerische Datenverarbeitung, Statistik und Probabilistik mit Instrumenten der Verpreisung und Verzinsung, der Ertragsrechnungen und der Kosten-Nutzen-Analysen (Haller et al. 2014, S. 14).

Unter dem Stichwort einer Internalisierung von externen Kosten haben sich hochrangige Kommissionen in der Bundesrepublik Deutschland oder auch in Frankreich für die Erweiterung der Berechnungsbasis des Bruttosozialprodukts eingesetzt. Mittlerweile ist das Konzept von buchhalterisch quantifizierbaren sogenannten „Umweltsystemdienstleistungen“ ein wichtiges Anliegen der internationalen Umweltpolitik. Ein Beispiel hierfür ist die Initiative WAVES. Die Abkürzung steht für ,Wealth 
Accounting and the Valuation of Ecosystem Services" und sollte gemäß der ersten Direktorin der Klimarahmenkonvention (UNFCCC), Christina Figueres, den weltpolitischen Erfolg, den die systematische Erfassung von Volkseinkommen im Bereich der Entwicklungspolitik hatte, auf die umweltpolitische Herausforderung übertragbar machen (Moreno et al. 2015, S. 27).

\section{Fazit: Verengungen durch die Zahlensicht}

Abschließend ist zu fragen, welcher Nutzen mit der diskursiven Einengung der Weltumwelt auf Zahlen verbunden gewesen ist, welche Kosten aufliefen und wer diese bezahlt. Die gängigen Quantifizierungen von $\mathrm{CO}_{2}$ und Biodiversität als Währungen der globalen Umweltpolitik haben verschiedene Wurzeln. Die numerische Kommunikation, die weltgesellschaftlichen Kommunikationszusammenhänge, die Umwelt als Thema und die Dominanz der Wirtschaftswissenschaften sind Aspekte, die ganz unterschiedliche historische Verläufe zeigen, aber in der gegenwärtigen Konstellation verschmelzen. Diese Prozesse sind vor dem Hintergrund der europäischen Expansion und des heute bestehenden Nord-Süd-Gegensatzes zu sehen. Denn noch immer unterscheiden sich Naturbezüge im Alltagsleben der meisten Menschen nach ihrer soziokulturellen und geografischen Herkunft. Man hat es überdies mit Prozessen der Kommodifizierung zu tun: So wie vor rund 350 Jahren der Boden zu einem handelbaren Gut wurde und später Debatten über das Wasser folgten, steht nun die Verwandlung von noch viel abstrakteren Größen an - die Verwandlung der Qualität der Atmosphäre und der Biosphäre in Objekte der kapitalistischen Dynamik.

Wenn man diese Dimensionen zu einer in sich stimmigen Geschichte verdichten will, wird rasch klar, dass die numerische Kommunikation nicht nur ein Ausdruck, sondern auch eine Ursache für die zu beobachtende globale Vergesellschaftung ist. Erst durch Zahlen wurde Umwelt zu einem weltgesellschaftlich kommunizierbaren Phänomen, d.h. erst nachdem lokale Wetterphänomene durch die Angabe von Temperaturgraden und Pegelständen zu isolierten, zählbaren Instanzen wurden, ähnlich wie Blumen im Herbarium oder $\mathrm{CO}_{2}$ in der Luft.

Die weltumweltpolitische Kommunikation befasst sich heute nicht nur fast ausschließlich mit der Klimafrage und der Biodiversität, sondern zeichnet sich in diesen Traditionslinien auch formal durch eine bemerkenswerte Fokussierung aus. Es geht hauptsächlich um folgende Dinge: Um das Verwandeln von „Natur“ in Listen von zählbaren Einheiten; um die Bewertung der Umweltwirkung von sozioökonomischen Umständen, um deren Vermessung in Zahlenform, um die Klassifikation und die Kategorisierung von unterschiedlichen Handlungsweisen und um dem Vergleich der sich darauf beziehenden Praktiken verschiedener Nationalstaaten und Unternehmen. Das alles geschah und geschieht in der Absicht, den Erdbewohnerinnen und Erdbewohnern die Chance auf ein gelingendes Leben zu sichern.

Die Quantifizierung der Weltumwelt bedeutet insgesamt die Verwandlung gesellschaftlicher Außenverhältnisse in buchhalterische Tabellen und Bilanzen. Es geht also - genaugenommen - um das Hereinholen des Außens in die gesellschaftlichen oder wirtschaftlichen Interaktionssysteme, und zwar global. Ironischerweise hat der 
Begriff „Umwelt“ mit dieser kommunikativen Bearbeitung seinen eigentlichen Sinn verloren, denn sie ist systemtheoretisch gerade keine Umwelt mehr, sondern einer der zentralsten Gegenstände weltgesellschaftlicher Selbstverständigung und damit selbst Teil eines Weltsystems.

Danksagung Für ihre konstruktive Kritik danke ich meiner Luzerner Kollegin Verena Halsmeyer und meinem Vater Andreas Speich sowie Sabine Höhler, Theresa Wobbe und Bettina Heintz.

Funding Open access funding provided by University of Luzern

Open Access Dieser Artikel wird unter der Creative Commons Namensnennung 4.0 International Lizenz veröffentlicht, welche die Nutzung, Vervielfältigung, Bearbeitung, Verbreitung und Wiedergabe in jeglichem Medium und Format erlaubt, sofern Sie den/die ursprünglichen Autor(en) und die Quelle ordnungsgemäß nennen, einen Link zur Creative Commons Lizenz beifügen und angeben, ob Änderungen vorgenommen wurden.

Die in diesem Artikel enthaltenen Bilder und sonstiges Drittmaterial unterliegen ebenfalls der genannten Creative Commons Lizenz, sofern sich aus der Abbildungslegende nichts anderes ergibt. Sofern das betreffende Material nicht unter der genannten Creative Commons Lizenz steht und die betreffende Handlung nicht nach gesetzlichen Vorschriften erlaubt ist, ist für die oben aufgeführten Weiterverwendungen des Materials die Einwilligung des jeweiligen Rechteinhabers einzuholen.

Weitere Details zur Lizenz entnehmen Sie bitte der Lizenzinformation auf http://creativecommons.org/ licenses/by/4.0/deed.de.

\section{Literatur}

Beckert, Sven. 2014. King Cotton. Eine Globalgeschichte des Kapitalismus. München: Beck.

Behrisch, Lars. 2016. Die Berechnung der Glückseligkeit. Statistik und Politik in Deutschland und Frankreich im späten Ancien Régime. Ostfildern: Thorbecke.

Bemmann, Martin. 2019. Internationale und Weltwirtschaftsstatistik. Beobachtungen, Überlegungen und Thesen zur Genese internationaler Wirtschaftsstatistik in den 1920er und 1930er Jahren. In Die Zählung der Welt. Kulturgeschichte der Statistik vom 18. bis 20. Jahrhundert, Hrsg. Stefan Haas, Michael C. Schneider und Nicolas Bilo, 195-217. Stuttgart: Franz Steiner.

Billig, Michael. 1995. Banal Nationalism. London: Sage.

Boenigk, Jens, und Sabina Wodniok. 2014. Biodiversität und Erdgeschichte. Berlin: Springer.

Borowy, Iris. 2014. Defining Sustainable Development for Our Common Future. A history of the World Commission on Environment and Development (Brundtland Commission). New York: United Nations.

Bürgi, Michael. 2004. Hinlänglich gebildet und republikanisch gesinnt. Meteorologie im bürgerlichen Verein. In Lokale Naturen. 150 Jahre Thurgauische Naturforschende Gesellschaft 1854-2004, Hrsg. Michael Bürgi und Daniel Speich, 37-62. Weinfelden: Wolfau.

Callon, Michel. 2009. Civilizing markets. Carbon Trading between in Vitro and in Vivo Experiments. Accounting. Organization and Society 34:456-482.

Campbell-Kelly, Martin, William Aspray, Nathan Ensmenger und Jeffrey R. Yost. 2016. Computer. A History of the Information Machine. Boulder: Westwiew Press.

Coase, Ronald. 1960. The Problem of Social Cost. Journal of Law and Economics 3:1-44.

Crosby, Alfred. 1991. Die Früchte des weissen Mannes. Ökologischer Imperialismus 900-1900. Frankfurt a. M.: Campus.

Daum, Andreas W. 2019. Alexander von Humboldt. München: Beck.

Demeritt, David. 2001. Scientific Forest Conservation and the Statistical Picturing of Nature's Limits in the Progressive-era United States. Environment and Planning D: Society and Space 19:431-459.

Devictor, Vincent, und Bernadette Bensaude-Vincent. 2016. From Ecological Records to Big Data. The Invention of Global Biodiversity. History and Philosophy of the Life Sciences 38(4):1-23.

Ehlers, Eckart. 2008. Das Anthropozän. Die Erde im Zeitalter des Menschen. Darmstadt: Wissenschaftliche Buchgesellschaft. 
Emane, Augustin. 2013. Nature en langues africaines du Gabon (Fang, Lingala Nzébi). In Tour du Monde des Concepts, Hrsg. Pierre Legendre, 191-195. Nantes: IEA.

Fatheuer, Thomas, Lili Fuhr und Barbara Unmüssig. 2015. Kritik der Grünen Ökonomie. München: Oekom.

Gissibl, Bernhard, Patrick Kupper und Sabine Höhler (Hrsg.). 2015. Civilizing Nature. National Parks in Global Historical Perspective. New York: Berghahn Books.

Graf, Rüdiger. 2019. Die Ökonomisierung der Umwelt und die Ökologisierung der Wirtschaft seit den 1970er Jahren. In Ökonomisierung. Debatten und Praktiken in der Zeitgeschichte, Hrsg. Rüdiger Graf, 188-214. Göttingen: Wallstein.

Gugerli, David. 2000. Wir wollen nicht im Trüben fischen. Gewässerschutz als Konvergenz von Bundespolitik, Expertenwissen und Sportfischerei (1950-1972). Schweizer Ingenieur und Architekt 118(13):281-290.

Haller, Lea, Sabine Höhler und Andrea Westermann. 2014. Einleitung. Rechnen mit der Natur. Ökonomische Kalküle um Ressourcen. Berichte zur Wissenschaftsgeschichte 37(1):8-19.

Harvey, David. 2005. A Brief History of Neoliberalism. Oxford: Oxford UP.

Heymann, Matthias. 2019. Klimakonstruktionen. Von der klassischen Klimatologie zur Klimaforschung. NTM 17:171-197.

Hironaka, Ann. 2014. Greening the Globe. World Society and Environmental Change. New York: Cambridge UP.

Höhler, Sabine. 2014. Von Biodiversität zu Biodiversifizierung. Eine Neue Ökonomie der Natur? Berichte zur Wissenschaftsgeschichte 37(1):60-77.

Höhler, Sabine. 2015. Spaceship Earth in the Environmental Age 1960-1990. London: Pickering \& Chatto.

Hopwood, Anthony G. 2009. Accounting and the Environment. Accounting, Organizations and Society 34:433-439.

Hünemörder, Kai F. 2005. 1972 - Epochenschwelle der Umweltgeschichte. In Natur- und Umweltschutz nach 1945. Konzepte, Konflikte, Kompetenzen, Hrsg. Franz-Josef Brüggemeier und Jens Ivo Engels, 125-144. Frankfurt a. M.: Campus.

Hupfer, Franziska. 2019. Regenmesser für den Staat. Niederschlagsstatistik und Anwendungserwartungen in der Schweiz (1860-1920). In Die Zählung der Welt. Kulturgeschichte der Statistik vom 18. bis 20. Jahrhundert, Hrsg. Stefan Haas, Michael C. Schneider und Nicolas Bilo, 73-97. Stuttgart: Franz Steiner.

Kaiser, Wolfram, und Jan-Henrik Meyer. 2017. Introduction. In International Organizations and Environmental Protection. Conservation and Globalization in the Twentieth Century, Hrsg. Wolfram Kaiser und Jan-Henrik Meyer, 1-29. New York: Berghahn.

Kramper, Peter. 2019. The Battle of the Standards. Messen, Zählen und Wiegen in Westeuropa 1660-1914. London: De Gruyter Oldenbourg.

Kuch, Declan. 2015. The Rise and Fall of Carbon Emissions Trading. Basingstoke: Palgrave Macmillan.

Kuchenbuch, David. 2012. Eine Welt. Globales Interdependenzbewusstsein und die Moralisierung des Alltags in den 1970er und 1980er Jahren. In Geschichte und Gesellschaft 38:158-184.

Kupper, Patrick. 2003. Die 1970er Diagnose. Grundsätzliche Überlegungen zu einem Wendepunkt der Umweltgeschichte. In Archiv für Sozialgeschichte 43:325-348.

Kupper, Patrick. 2012. Wildnis schaffen. Eine transnationale Geschichte des Schweizerischen Nationalparks. Bern: Haupt.

Lucht, Michael, und Gorden Spangardt. Hrsg. 2005: Emissionshandel. Ökonomische Prinzipien, rechtliche Regelungen und technische Lösungen für den Klimaschutz. Berlin: Springer.

Macekura, Stephen. 2015. Of Limits and Growth. The Rise of Global Sustainable Development in the Twentieth Century. New York: Cambridge UP.

MacKenzie, Donald. 2009. Making Things the Same. Gases, Emission Rights and the Politics of Carbon Markets. Accounting, Organization and Society 34:440-455.

Macpherson, Crawford B. 1962. The Political Theory of Possessive Individualism. Hobbes to Locke. Oxford: Clarendon Press.

McCormick, John. 1989. Reclaiming Paradise. The Global Environmental Movement. Indianapolis, Indiana UP.

McNeill, John R. 2000. Blue Planet. Die Geschichte der Umwelt im 20. Jahrhundert. Frankfurt a. M.: Campus.

Meckling, Jonas. 2011. Carbon Coalitions. Business, Climate Politics, and the Rise of Emissions Trading. Boston: MIT Press.

Meyer, John W., David John Frank, Ann Hironaka, Evan Schofer und Nancy Brandon Tuma. 2005. Die Entstehung eines globalen Umweltschutzregimes von 1870 bis 1990 . In John W. Meyer, Weltkultur. 
Wie die westlichen Prinzipien die Welt durchdringen, Hrsg. Georg Krücken, 235-299. Frankfurt a. M.: Suhrkamp.

Moreno, Camila, Lili Fuhr und Daniel Speich Chassé. 2015. Carbon Metrics. Global Abstractions and Ecological Epistemicide. Berlin: Heinrich Böll Stiftung.

Ogle, Vanessa. 2015. The Global Transformation of Time 1870-1950. Cambridge MA: Harvard UP.

Pfister, Christian (Hrsg.). 1995. Das 1950er-Syndrom. Der Weg in die Konsumgesellschaft. Bern: Haupt.

Ptak, Ralf. 2008. Grundlagen des Neoliberalismus. In Kritik des Neoliberalismus, Hrsg. Christoph Butterwegge et al., 13-88. Wiesbaden: VS Verlag für Sozialwissenschaft.

Radkau, Joachim. 2000. Natur und Macht. Eine Weltgeschichte der Umwelt. München: Beck.

Radkau, Joachim. 2011. Die Ära der Ökologie. Eine Weltgeschichte. München: Beck.

Radkau, Joachim, und Frank Uekötter. Hrsg. 2003. Naturschutz und Nationalsozialismus. Frankfurt a. M.: Campus.

Rahmstorf, Stefan, und Hans Joachim Schellnhuber. 2019. Der Klimawandel. Diagnose, Prognose, Therapie. München: Beck.

Rausch, Heribert: Ungewisse Erfolgsaussichten der Umweltkonferenz. In: NZZ 09.06.1972

Rausch, Heribert: Positive Bilanz der Stockholmer Umweltkonferenz. In: NZZ 19.06.1972

Renn, Jürgen, und Bernd Scherer. Hrsg. 2015. Das Anthropozän. Zum Stand der Dinge. Berlin: Matthes \& Seitz.

Robertson, Morgan M. 2006. The Nature that Capital Can See. Science, State, and Market in the Commodification of Ecosystem Services. In Environment and Planning D: Society and Space 24:367-387.

Ruppel, Sophie. 2019. Botanophilie. Mensch und Pflanze in der aufklärerisch-bürgerlichen Gesellschaft um 1800. Göttingen: Vandenhoeck \& Ruprecht.

Schleper, Simone. 2019. Planning for the Planet. Environmental Expertise and the International Union for the Conservation of Nature and Natural Resources 1960-1980. New York: Berghahn.

Schmelzer, Matthias. 2016. The Hegemony of Growth. The OECD and the Making of the Economic Growth Paradigm. New York: Cambridge UP.

Schneider, Ute. 2004. Die Macht der Karten. Eine Geschichte der Kartographie vom Mittelalter bis heute. Darmstadt: Wissenschaftliche Buchgesellschaft.

Schwarzenbach, Alexis. 2011. WWF. Die Biografie. München: Heyne.

Seibel, Benjamin. 2016. Cybernetic Government. Informationstechnologie und Regierungsrationalität von 1943-1970. Wiesbaden: Springer.

Selcer, Perrin. 2018. The Post-War Origins of the Global Environment. How the United Nations Built Spaceship Earth. New York: Columbia UP.

Steinmetz, Willibald (Hrsg.). 2019. The Force of Comparison. A New Perspective on Modern European History and the Contemporary World. New York: Berghahn.

Strasser, Bruno. 2012. Data-Driven Sciences. From Wonder Cabinets to Electronic Databases. Studies in History and Philosophy of Biological and Biomedical Sciences 43:85-87.

Wallerstein, Immanuel. 1986. Das moderne Weltsystem. Frankfurt a. M.: Syndikat.

Ward, Barbara, und René Dubos. 1972. Wie retten wir unsere Erde? Umweltschutz. Bilanz und Prognose. Basel: Herder.

Warde, Paul, Libby Robin und Sverker Sörlin. 2019. The Environment. A History of the Idea. Baltimore: Johns Hopkins UP.

WHO 2005. Millennium Ecosystem Assessment. Ecosystems and Human Well-Being. Genf: WHO.

Daniel Speich Chassé 1969, Dr. phil., Professor für Globalgeschichte, Universität Luzern. Forschungsgebiete: Globalgeschichte, Umweltgeschichte, Geschichte des ökonomischen Wissens, Geschichte der numerischen Kommunikation und der Digitalisierung. Veröffentlichungen: Die Erfindung des Bruttosozialprodukts. Globale Ungleichheit in der Wissensgeschichte der Ökonomie. Göttingen 2013; In Search of a Global Centre of Calculation. The Washington Statistical Conferences of 1947, in: W. Steinmetz (Hrg.): The Force of Comparison. A New Perspective on Modern European History and the Contemporary World. New York 2019. 